\title{
Flow Instabilities in Mechanically Agitated Stirred Vessels
}

\author{
Chiara Galletti and Elisabetta Brunazzi \\ Department of Chemical Engineering, \\ Industrial Chemistry and Materials Science, University of Pisa
}

Italy

\section{Introduction}

A detailed knowledge of the hydrodynamics of stirred vessels may help improving the design of these devices, which is particularly important because stirred vessels are among the most widely used equipment in the process industry.

In the last two decades there was a change of perspective concerning stirred vessels. Previous studies were focused on the derivation of correlations able to provide global performance indicators (e.g. impeller flow number, power number and mixing time) depending on geometric and operational parameters. But recently the attention has been focused on the detailed characterization of the flow field and turbulence inside stirred vessels (Galletti et al., 2004a), as only such knowledge is thought to improve strongly the optimization of stirred vessel design.

The hydrodynamics of stirred vessels has resulted to be strongly three dimensional, and characterised by different temporal and spatial scales which are important for the mixing at different levels, i.e. micro-mixing and macro-mixing.

According to Tatterson (1991) the hydrodynamics of a mechanically agitated vessel can be divided at least into three flow systems:

- impeller flows including discharge flows, trailing vortices behind the blades, etc.;

- wall flows including impinging jets generated from the impeller, boundary layers, shed vortices generated from the baffles, etc.;

- $\quad$ bulk tank flows such as large recirculation zones.

Trailing vortices originating behind the impeller blades have been extensively studied for a large variety of impellers. For instance for a Rushton turbine (RT) they appear as a pair, behind the lower and the upper sides of the impeller blade, and provide a source of turbulence that can improve mixing. Assirelli et al. (2005) have shown how micro-mixing efficiency can be enhanced when a feeding pipe stationary with the impeller is used to release the fed reactant in the region of maximum dissipation rate behind the trailing vortices. Such trailing vortices may also play a crucial role in determining gas accumulation behind impeller blades in gas-liquid applications, thus affecting pumping and power dissipation capacity of the impeller.

But in the last decade lots of investigations have pointed out that there are other important vortices affecting the hydrodynamics of stirred vessels. In particular it was found that the flow inside stirred vessels is not steady but characterised by different flow instabilities, 
which can influence the flow motion in different manners. Their knowledge and comprehension is still far from complete, however the mixing optimisation and safe operation of the stirred vessel should take into account such flow variations.

The present chapter aims at summarizing and discussing flow instabilities in mechanically agitated stirred vessels trying to highlight findings from our research as well as from other relevant works in literature. The topic is extremely wide as flow instabilities have been detected with different investigation techniques (both experimental and numerical) and analysis tools, in different stirred vessel/impeller configurations.

Thus investigation techniques and related analysis for the flow instability detection will be firstly overviewed. Then a possible classification of flow instabilities will be proposed and relevant studies in literature will be discussed. Finally, examples of findings on different flow instabilities and their effects on the mixing process will be shown.

\section{Investigation techniques}

Researchers have employed a large variety of investigation techniques for the detection of flow instabilities. As such techniques should allow identifying flow instabilities, they should be able to detect a change of the flow field (or other relevant variables) with time. Moreover a good time resolution is required to allow an accurate signal processing. Regarding this point, actually flow instabilities in stirred vessels are generally low frequencies phenomena as their frequency is much smaller than the impeller rotational frequency N; so, effectively, the needed temporal resolution is not so high. Anyway the acquisition frequency should at least fulfil the Nyquist criterion.

The graph of Fig. 1a summarises the main techniques, classified as experimental and numerical, employed so far for the investigation of flow instabilities. A brief description of the techniques will be given in the following text in order to highlight the peculiarities of their applications to stirred vessels.

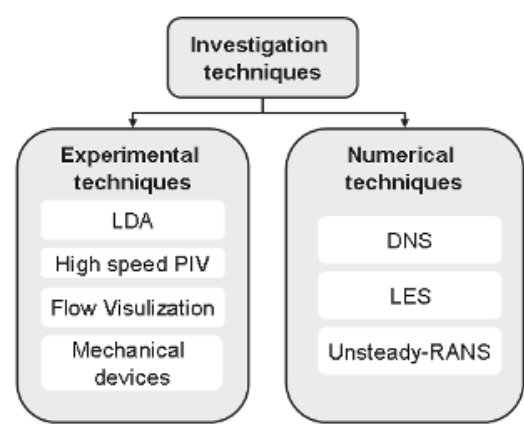

(a)

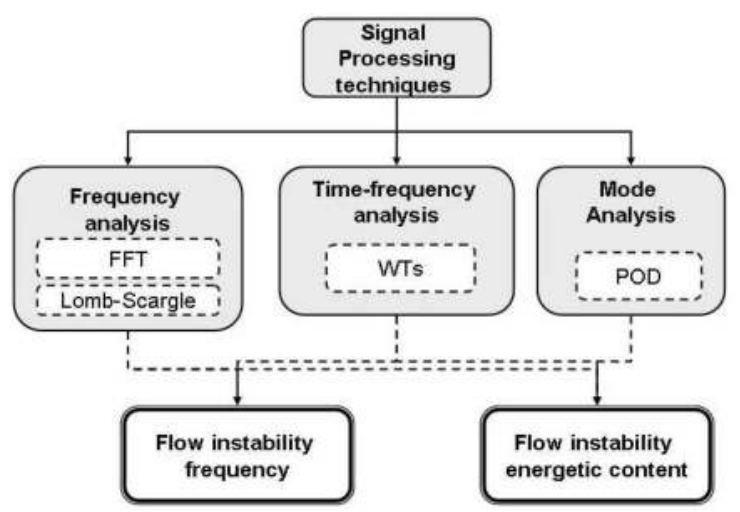

(b)

Fig. 1. Overview of investigation (a) and analysis (b) techniques for flow instability characterization in stirred vessels.

\subsection{Experimental techniques}

Laser Doppler anemometry (LDA) is one of the mostly used experimental technique for flow instability detection. LDA is an optical non-intrusive technique for the measurement of 
the fluid velocity. It is based on the Doppler shift of the light scattered from a 'seeding' particle, which is chosen to be nearly neutrally buoyant and to efficiently scatter light. LDA does not need any calibration and resolves unambiguously the direction of the velocity. Moreover it provides high spatial and temporal resolutions. These are very important for flow instability detection. In addition, more than one laser Doppler anemometer can be combined to perform multi-component measurements. The application of LDA to cylindrical stirred vessels requires some arrangement in order to minimize refraction effects at the tank walls, so often the cylindrical vessel is placed inside a square trough.

Particle Image Velocimetry (PIV) is also an optical technique which allows the velocity of a fluid to be simultaneously measured throughout a region illuminated by a two-dimensional light sheet, thus enabling the instantaneous measurements of two velocity components. However recently the use of a stereoscopic approach allows all three velocity components to be recorded. So far the temporal resolution of PIV measurements has been limited because the update rate of velocity measurements, governed by the camera frame rate and the laser pulse rate, was too low. Thus PIV was not suited for the investigation of flow instabilities. However recently, high-frame rate PIV systems have been developed allowing flow measurements with very high update rates (more than $10 \mathrm{kHz}$ ); thus its use for the analysis of flow instabilities in stirred vessels has been explored by some investigators. Similarly to LDA, also PIV requires the fluid and vessel walls to be transparent as well as actions to minimize refraction effects at the tank curvature.

Different flow visualization techniques have also been used to help clarifying the mechanism of flow instabilities. Such flow visualization techniques may simply consist of tracing the fluid with particles and recording with a camera a region of the flow illuminated by a laser sheet. More sophisticated techniques are able of providing also concentration distribution: for example Laser Induced Fluorescence, LIF, uses a fluorescent marker and a camera (equipped with a filter corresponding to the wave of fluorescence) which detects the fluorescence levels in the liquid.

In addition to such optical instruments, different mechanical devices have been used in literature for the detection of flow instabilities. Such devices are based on the measurements of the effect of flow instabilities on some variables. Bruha et al. (1995) employed a "tornadometer", that is a device which allows measuring the temporal variation of the force acting on a small target placed into the flow where instabilities are thought to occur. Paglianti et al. (2006) proved that flow instabilities in stirred vessels could be detected by pressure transducers positioned at the tank walls. The pressure transducers provided time series of pressure with a temporal resolution suited for the flow instability detection. Such a technique is particularly interesting as it is well suited for industrial applications. Haam et al. (1992) identified flow instabilities from the measurement of heat flux and temperature at the walls through heat flux sensors and thermocouples. Hasal et al. (2004) measured the tangential force acting on the baffles as a function of time by means of mechanical devices. Also power number measurements (as for instance through strain gauge techniques) have been found to give an indication of flow instabilities related to change in the circulation loop (Distelhoff et al., 1995).

\subsection{Numerical techniques}

Numerical models have also been used for the investigation of flow instabilities in stirred vessels, especially because of the increasing role of Computational Fluid Dynamics (CFD). Logically, since the not steady nature of such instabilities, transient calculation techniques 
have to be employed. These may be classified in: Unsteady Reynolds-averaged NavierStokes equations (URANS), Large Eddy Simulation (LES) and Direct Numerical Simulation (DNS)

URANS employs the usual Reynolds decomposition, leading to the Reynolds-averaged Navier-Stokes equations, but with the transient (unsteady) term retained. Subsequently the dependent variables are not only a function of the space coordinates, but also a function of time. Moreover, part of the turbulence is modelled and part resolved. URANS have been applied to study stirred vessels by Torré et al. (2007) who found indications on the presence of flow instabilities from their computations; however their approach was not able to identify precessional flow instabilities.

LES consists of a filtering operation, so that the Navier-Stokes are averaged over the part of the energy spectrum which is not computed, that is over the smaller scales. Since the remaining large-scale turbulent fluctuations are directly resolved, LES is well suited for capturing flow instabilities in stirred vessels, although it is very computationally expensive. This has been shown for both single-phase (for example Roussinova et al., 2003, Hartmann et al., 2004, Nurtono et al. 2009) and multi-phase (Hartmann et al., 2006) flows.

DNS consists on the full resolution of the turbulent flow field. The technique has been applied by Lavezzo et al. (2009) to an unbaffled stirred vessel with $\operatorname{Re}=1686$ providing evidence of flow instabilities.

\section{Analysis techniques}

The above experimental or modelling investigations have to be analysed with suited tools in order to get information on flow instabilities. These consist mainly of signal processing techniques, which are applied to raw data, such as LDA recordings of the instantaneous velocity, in order to gain information on the characteristics of flow instabilities.

Two kinds of information have been extracted so far:

- frequency of the flow instabilities as often they appear as periodic phenomena;

- relevance of flow instabilities on the flow motion.

Among the techniques which have been employed in literature for the characterization of flow instabilities in stirred vessels, there are (see Fig. 1b):

- frequency analysis techniques (the Fast Fourier Transforms and the Lomb-Scargle periodogram method);

- time-frequency analysis techniques (Wavelet Transforms);

- principal component analysis (Proper Orthogonal Decomposition).

Whereas the first two techniques have been largely used for the determination of the flow instability frequency, the latter has been used to evaluate the impact of flow instabilities on the motion through the analysis of the most energetic modes of the flow.

\subsection{Frequency analysis}

The Fast Fourier Transform (FFT) decomposes a signal in the time domain into sines and cosines, i.e. complex exponentials, in order to evaluate its frequency content. Specifically the FFT was developed by Cooley \& Tukey (1965) to calculate the Fourier Transform of a K samples series with $\mathrm{O}\left(\mathrm{K}_{\left.\log _{2} \mathrm{~K}\right)}\right.$ operations. Thus FFT is a powerful tool with low computational demand, but it can be performed only over data evenly distributed in time. In case of LDA recordings, these should be resampled and the original raw time series replaced with series uniform in time. As for the resampling techniques, simple methods like 
the "Nearest Neighbour" or the "Sample and Hold" should be preferred over complex methods (e.g. "Linear Interpolation", "Spline Interpolation"), because the latter bias the variance of the signal. It should be noticed that the resampled series contains complete information about the spectral components up to the Nyquist critical frequency $f c=1 / 2 \Delta$ where $\Delta$ in the sampling interval. At frequencies larger than the Nyquist frequency the information on the spectral components is aliased.

The Lomb-Scargle Periodogram (LSP) method (Lomb, 1976, Scargle, 1982) performs directly on unevenly sampled data. It allows analysing frequency components larger than the Nyquist critical frequency: this is possible because in irregularly spaced series there are a few data spaced much closer than the average sampling rate, removing ambiguity from any aliasing. The method is much more computational expensive than FFTs, requiring $\mathrm{O}\left(10^{2} \mathrm{~K}^{2}\right)$ operations.

It is worthwhile discussing the suitability of the analysis techniques described above for the investigation of flow instabilities and what are the main parameters to be considered. Flow instabilities are low frequency phenomena, therefore we are interested in the low frequency region of the frequency spectrum. The lowest frequency which can be resolved with both the FFT and Lomb-Scargle method is inversely related to the acquisition time; hence longer sampling times yield better frequency resolutions. This explains the long observations made for flow instabilities detection in stirred vessels. In our works on flow instabilities we have used typically LDA recordings at least $800 \mathrm{~s}$ long. In other words the sampling time should be long enough to cover a few flow instabilities cycles. As the time span covered by a series is proportional to the number of samples, the application of the LSP to long series requires strong computational effort.

A benchmark between the two methods is provided in Galletti (2005) and shown in Fig. 2.

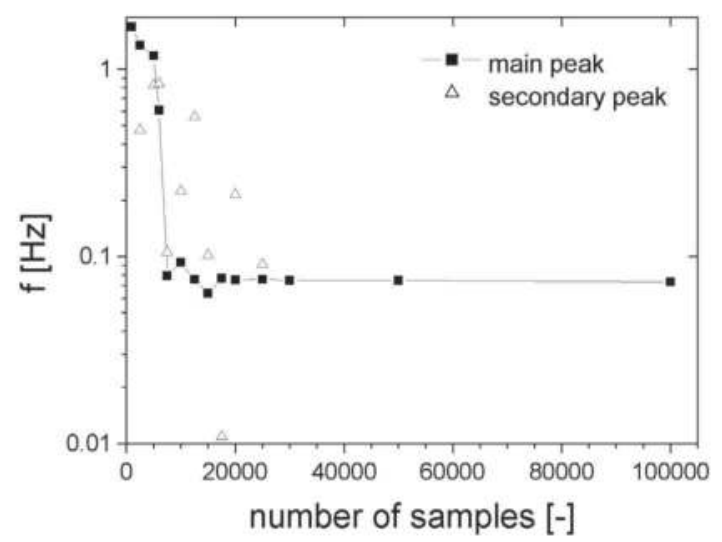

Fig. 2. Frequency of the main and the secondary peak in the low frequency region of the spectrum calculated with the Lomb-Scargle method as a function of the number of samples. $\mathrm{RT}, \mathrm{D} / \mathrm{T}=0.33, \mathrm{C} / \mathrm{T}=0.5, \mathrm{Re}=27,000$. Galletti (2005).

The solid squares show the frequency $f$ of the main peak identified in the spectrum calculated with the LSP as function of the number of samples used for the analysis. It can be observed that $\mathrm{f}$ is scattered for low numbers of samples, and it approaches asymptotically the value of $\mathrm{f}$ $=0.073 \mathrm{~Hz}$ (the same of the FFT analysis over the whole acquisition time of $800 \mathrm{~s}$ with 644,000 
samples) as the number of samples increases. The empty triangles indicate the presence of further low frequency peaks. The main fact to be aware of is that low time intervals conceal the flow instabilities by covering only a portion of the fluctuations.

\subsection{Time-frequency analysis}

Both FFT and LSP inform how much of each frequency component exists in the signal, but they do not tell us when in time these frequencies occur in the signal. For transient flows it may be of interest the time localisation of the spectral component. The Wavelet Transform (WT) is capable of providing the time and frequency information simultaneously, hence it gives a time-frequency representation of the signal (Daubechies, 1990, Torrence and Compo, 1998). The WT breaks the signal into its "Wavelets", that are functions obtained from the scaling and the shifting of the "mother Wavelet" $\psi$. The WT has been proposed for the investigation of stirred vessels by Galletti et al. (2003) and subsequently applied by Roy et al. (2010).

\subsection{Proper orthogonal decomposition}

POD is a linear procedure, based on temporal and spatial correlation analysis, which allows to decompose a set of signals into a modal base, with the first mode being the most energetic (related to large-scale structures thus trailing vortices and flow instabilities) and the last being the least energetic (smaller scales of turbulence). It was first applied for MI characterisation by Hasal et al. (2004) and latterly by Ducci \& Yianneskis (2007). An in-depth explanation of the methodology is given in Berkooz et al. (1993).

\section{Classification of flow instabilities}

A possible classification of flow instabilities in stirred vessels is reported in Fig. 3. The graph is not aimed at imposing a classification of flow instabilities, however it suggests a way of interpretation which may be regarded as a first effort to comprehend all possible instabilities.

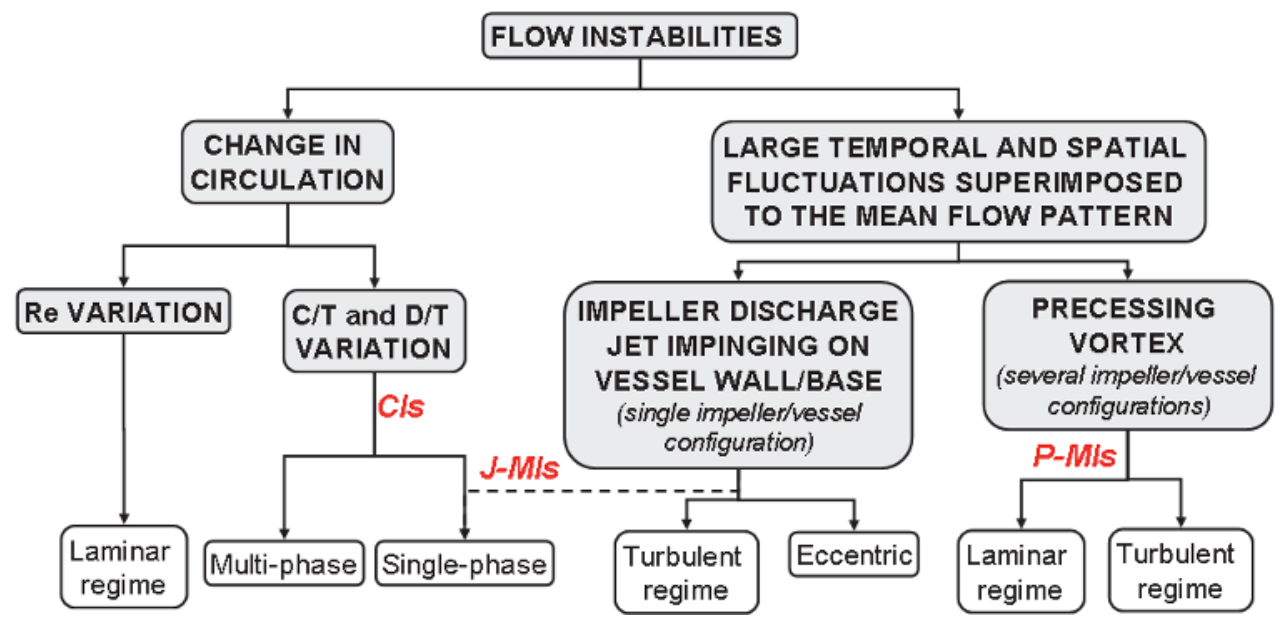

Fig. 3. Possible classification of flow instabilities in stirred vessels. 


\subsection{Change in circulation pattern}

A first kind of flow instability (see left-hand side of the diagram of Fig. 3) manifests as a real change in the circulation pattern inside the tank. Two main sources of such a change have been identified: a variation of the Reynolds number (Re) or a variation of the impeller/vessel geometrical configuration.

In relation to the former source, Nouri \& Whitelaw (1990) reported a transition due to Re variations in the flow pattern induced by a $60^{\circ} \mathrm{PBT}$ with $\mathrm{D}=\mathrm{T} / 3$ set at $\mathrm{C}=\mathrm{T} / 3$ in a vessel of $\mathrm{T}=0.144 \mathrm{~m}$. For non-Newtonian fluids a flow pattern transition from a radial to an axial flow was observed as the $\operatorname{Re}$ was increased up to $\operatorname{Re}=4,800$. For Newtonian fluids the authors observed that the flow pattern transition occurred at about $\operatorname{Re}=650$. This value was also confirmed by the power number measurements through strain-gauges carried out by Distelhoff et al. (1995). Similar investigations on such transition may be found in the works of Hockey (1990) and Hockey \& Nouri (1996).

Schäfer et al. (1998) observed by means of flow visualisation the flow discharged by a $45^{\circ}$ PBT to be directed axially at higher Re and radially at lower Re. The flow stream direction was unstable, varying from radial to axial, for $\mathrm{Re}=490-510$. A similar flow transition was also indicated by Bakker et al. (1997) who predicted with CFD techniques the flow pattern generated from a 4-bladed $45^{\circ} \mathrm{PBT}$ of diameter $\mathrm{D}=\mathrm{T} / 3$ and set at $\mathrm{C}=\mathrm{T} / 3$ inside a tank of $\mathrm{T}$ $=0.3 \mathrm{~m}$. The regime was laminar, the Reynolds number being varied between 40 and 1,200. The impeller discharge stream was directed radially for low Re numbers, however for Re larger than 400 the flow became more axial, impinging on the vessel base rather than on the walls.

A second source of instabilities, manifesting as a flow pattern change, is associated with variations of the impeller/vessel geometrical configuration, which means either variations of the distance of the impeller from the vessel bottom $(\mathrm{C} / \mathrm{T})$ or variation of the impeller diameter $(\mathrm{D} / \mathrm{T})$ or a combination of both variations.

This kind of instabilities were firstly noticed by Nienow (1968) who observed a dependency on the clearance of the impeller rotational speed required to suspend the particles $(\mathrm{Njs})$ in a solid-liquid vessel equipped with a $\mathrm{D}=0.35 \mathrm{~T}$ RT. He observed that for $\mathrm{C}<\mathrm{T} / 6$ the pattern was different (the discharge stream was directed downwards towards the vessel corners) from the typical radial flow pattern, providing low Njs values. Baldi et al. (1978) also observed a decrease of the Njs with the impeller off-bottom clearance for a 8-bladed turbine. Conti et al. (1981) found a sudden decrease of the power consumption associated with the change in the circulation pattern when lowering the impeller clearance of a 8-bladed turbine. The aforementioned authors concluded that the equation given by Zwietering (1958) for the calculation of the Njs should be corrected in order to take into account the dependency on $\mathrm{C} / \mathrm{T}$.

The dependency of the power number on the impeller off-bottom clearance was also observed by Tiljander \& Theliander (1993), who measured the power consumption of two PBT of different sizes, i.e. $\mathrm{D}=\mathrm{T} / 3$ and $\mathrm{D}=\mathrm{T} / 2$, and a high flow impeller of $\mathrm{D}=\mathrm{T} / 2$. The visual observation of the flow pattern revealed that at the transition point between the axial and the radial flow patterns, the circulation inside the vessel appears chaotic.

Ibrahim \& Nienow (1996) investigated the efficiency of different impellers, i.e. a RT, a PBT pumping either upwards or downwards, a Chemineer HE3 and a Lightnin A310 hydrofoil pumping downwards and a Ekato Intermig agitator, for solids suspension. For the RT, the aforementioned authors observed a sudden decrease of both the impeller speed and the mean dissipation rate required to just suspend the particles as the clearance was decreased 
from $C=T / 3$ down to $C=T / 6$ for the impeller having $D=T / 3$; such a clearance corresponded to the transition from the radial flow pattern to the axial.

Subsequently, a strong influence of the clearance on the suspension of particles was confirmed also by Myers et al. (1996) for three axial impellers. If the clearance was sufficiently high the discharge flow impinged on the vessel wall rather then the base, leading to a secondary circulation loop which was directed radially inward at the vessel base and returned upwards to the impeller at the centre of the vessel. Such a reversed flow occurred for $C>0.45 \mathrm{~T}$ for a PBT of diameter $\mathrm{D}=0.41 \mathrm{~T}$ and for $\mathrm{C}>0.25 \mathrm{~T}$ for a straight-blade turbine of the same diameter, whereas only for very high clearances $(C>0.95 T)$ for a high efficiency Chemineer impeller having the same diameter.

Bakker et al. (1998) reported that the flow pattern generated by either a PBT or a three-blade high efficiency impeller depended on $\mathrm{C} / \mathrm{T}$ and $\mathrm{D} / \mathrm{T}$, influencing the suspension of the particles.

Armenante \& Nagamine (1998) determined the Njs and the power consumption of four impellers set at low off-bottom clearances, typically $\mathrm{C}<\mathrm{T} / 4$. For radial impellers, i.e. a RT and a flat blade turbine, they observed that the clearance at which the change in the flow pattern from a radial to an axial type occurred was a function of both impeller type and size, i.e. $\mathrm{D} / \mathrm{T}$. In particular the flow pattern changed at lower $\mathrm{C} / \mathrm{T}$ for larger impellers. This was in contrast with previous works (see for example Conti et al., 1981) which reported a clearance of transition independent on D/T. For instance Armenante \& Nagamine (1998) found the flow pattern transition to occur at $0.16<\mathrm{C} / \mathrm{T}<0.19$ for a Rushton turbine with a diameter $\mathrm{D}=0.217 \mathrm{~T}$ and at $0.13<\mathrm{C} / \mathrm{T}<0.16$ for a $\mathrm{D}=0.348 \mathrm{~T}$ RT. For the flat blade turbine the clearances at which the transition took places were higher, being of 0.22-0.24 and 0.190.21 for the two impeller sizes $\mathrm{D}=0.217 \mathrm{~T}$ and $\mathrm{D}=0.348 \mathrm{~T}$, respectively.

Sharma \& Shaikh (2003) provided measurements of both Njs and power consumption of solids suspension in stirred tanks equipped with $45^{\circ}$ PBT with 4 and 6 blades. They plotted the critical speed of suspension Njs as a function of $\mathrm{C} / \mathrm{T}$ distinguishing three regions, according to the manner the critical suspension speed varied with the distance of the impeller from the vessel base. As the impellers were operating very close to the vessel base, the Njs was observed to be constant with $\mathrm{C} / \mathrm{T}$ (first region); then for higher clearances Njs increased with $\mathrm{C} / \mathrm{T}$ because the energy available for suspension decreased when increasing the distance of the impeller from the vessel base (second region), and finally (third region) for high clearances the Njs increased with $\mathrm{C} / \mathrm{T}$ with a slope higher than that of the second region. The onset of third region corresponded to the clearance at which the flow pattern changed from the axial to the radial flow type. In addition the aforementioned authors observed that as the flow pattern changed the particles were alternatively collected at the tank base in broad streaks and then suddenly dispersed with a certain periodicity. They concluded that a kind of instabilities occurred and speculated that maybe the PBT behaved successively as a radial and axial flow impellers.

The influence of $\mathrm{C}$ on the flow pattern has been intensively studied also for single-phase flow in stirred tanks. Yianneskis et al. (1987) showed that the impeller off-bottom clearance affects the inclination of the impeller stream of a Rushton turbine of diameter $D=T / 3$. In particular the discharge angle varied from $7.5^{\circ}$ with respect to the horizontal plane for $\mathrm{C}=$ $\mathrm{T} / 4$ down to $2.5^{\circ}$ for $\mathrm{C}=\mathrm{T} / 2$.

Jaworski et al. (1991) measured with LDA the flow patterns of a 6-bladed $45^{\circ} \mathrm{PBT}$ having a diameter $\mathrm{D}=\mathrm{T} / 3$ for two impeller clearances, $\mathrm{C}=\mathrm{T} / 4$ and $\mathrm{C}=\mathrm{T} / 2$. For the lower impeller clearance, the impeller stream impinged on the vessel base and generated an intensive radial 
circulation from the vessel axis towards the walls. For the higher clearance the impeller stream turned upwards before reaching the base of the vessel, generating also a reverse flow directed radially from the walls towards the vessel axis at the base of the vessel.

Kresta \& Wood (1993) measured the mean flow field of a vessel stirred with a 4-bladed $45^{\circ}$ PBT for two impeller sizes, i.e. $\mathrm{D}=\mathrm{T} / 3$ and $\mathrm{D}=\mathrm{T} / 2$, and varying the impeller clearance systematically from $\mathrm{T} / 20$ up to $\mathrm{T} / 2$. They observed that the circulation pattern underwent a transition at $C / D=0.6$, and for the larger impeller $(D=T / 2)$ such a transition was accompanied by a deflection of the inclination of the discharge stream toward the horizontal.

Ibrahim \& Nienow (1995) measured the power number of different impellers for a wide range of Reynolds number, i.e. $40<\mathrm{Re}<50,000$, in Newtonian fluids. For a $\mathrm{D}=\mathrm{T} / 3 \mathrm{RT}$ they observed that the power numbers with clearances of $\mathrm{C}=\mathrm{T} / 3$ and $\mathrm{C}=\mathrm{T} / 4$ was the same for all Re; however for $\mathrm{C}=\mathrm{T} / 6$ the discharge flow was axial rather than radial and the associated power number was considerably lower (by about $25 \%$ ) for all the range of $\mathrm{Re}$ investigated. For a $\mathrm{D}=\mathrm{T} / 2 \mathrm{RT}$ a radial discharge flow was still observed at $\mathrm{C}=\mathrm{T} / 6$ for all Re except for those with the highest viscosity (1 Pa.s).

Rutherford et al. (1996a) investigated the flow pattern generated by a dual Rushton impeller and observed different circulation patterns depending on the impeller clearance of the lower impeller and the separations between the two impellers, observing three stable flow patterns: "parallel flow", "merging flow" and "diverging flow" patterns.

Mao et al. (1997) measured with LDA the flow pattern generated from various PBT of different sizes in the range of $0.32<\mathrm{D} / \mathrm{T}<0.6$ and number of blades varying from 2 to 6 in a stirred vessel in turbulent regime $(\operatorname{Re}>20,000)$. They used two impeller off-bottom clearances, $\mathrm{C}=\mathrm{T} / 3$ and $\mathrm{C}=\mathrm{T} / 2$, observing a secondary circulation loop with the higher clearance.

Montante et al. (1999) provided a detailed investigation of the flow field generated by $\mathrm{D}=$ $\mathrm{T} / 3 \mathrm{RT}$ placed at different off-bottom clearances varying from $\mathrm{C}=0.12 \mathrm{~T}$ to $\mathrm{C}=0.33 \mathrm{~T}$. They found that the conventional radial flow pattern (termed "double-loop" pattern) occurred for $\mathrm{C}=0.20 \mathrm{~T}$, but it was replaced by an axial flow pattern (termed "single loop" pattern) as the clearance was decreased to $C=0.15 \mathrm{~T}$. A reduction of the power number from $4.80-4.85$ for $\mathrm{C} / \mathrm{T}=0.25-0.33$ down to 3.80 as the clearance was decreased to $\mathrm{C} / \mathrm{T}=0.12-0.15$ was reported, so that the power consumption was reduced by about $30 \%$ as the flow underwent a transition from the double- to the single-loop pattern.

\subsubsection{Clearance instabilities (Cls)}

Galletti et al. (2003, 2005a, 2005b) studied the flow pattern transition for a D = T/3 RT and identified a kind of flow instabilities, which will be denoted as CIs (clearance instability). The authors found that the flow pattern transition (single- to double-loop pattern) occurred for $\mathrm{C} / \mathrm{T}=0.17-0.2$, thus within an interval of clearances of about $0.03 \mathrm{~T}$. Such $\mathrm{C}$ interval was dependent on the fluid properties, lower clearances being observed for more viscous fluids. At clearances of flow pattern transition the velocity time series indicated flow pattern instabilities as periods of double-loop regime, single-loop regime and "transitional" state that followed each other. When the flow underwent a change from one type of circulation to another, the transitional state was always present and separated in time the single- from the double-loop flow regime. Nevertheless, a flow pattern could change firstly into the transitional state and afterwards revert to the original flow regime, without changing the type of circulation. The occurrence of the three flow regimes was shown to be random, and 
their lifetimes could be significant, often of the order of few minutes. The time duration of the three flow regimes depended on the impeller clearance, higher clearances promoting the double-loop regime. Moreover the time duration of the three flow regimes depended on the impeller rotational speed, higher impeller rotational speeds promoting the double-loop regime.

An example of flow pattern transition is shown in the LDA time series of Fig. 4a which indicated different regimes, that can be attributed to the double-loop, transitional and single-loop patterns. The most surprising finding was that within the transitional state an instability was manifested as a periodic fluctuation of the flow between the double and the single-loop regimes, characterised by a well-defined frequency f. Such frequency was linearly dependent on the impeller speed according to $f^{\prime}=f / N=0.12$.

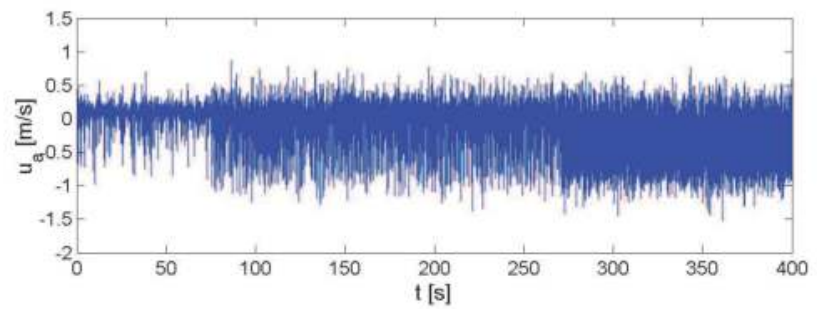

(a)

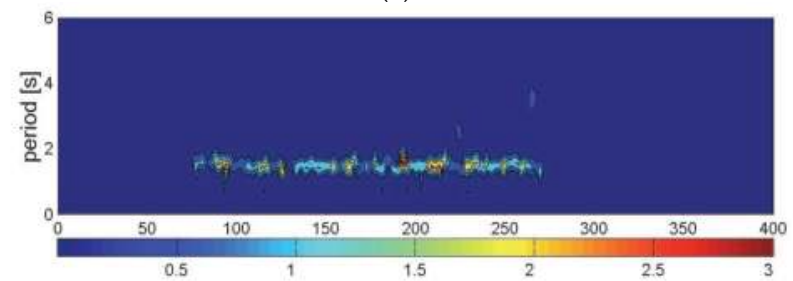

(b)

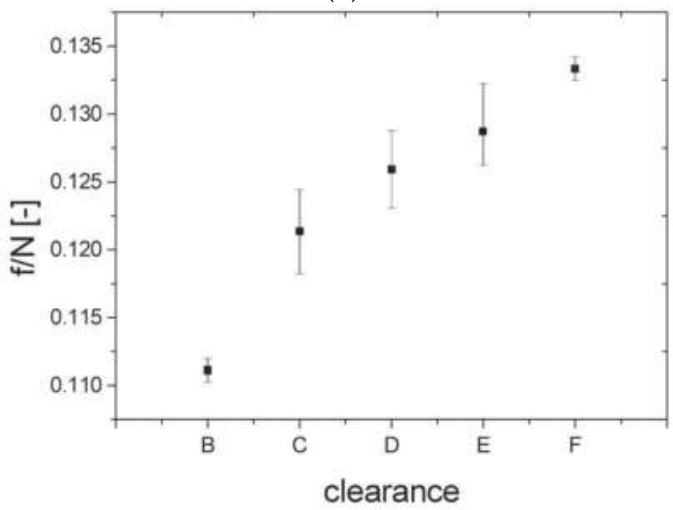

(c)

Fig. 4. Wavelet power analysis of axial velocity data: (a) time series; (b) Wavelet power spectrum; (c) dependence of frequency on impeller clearance ( $\mathrm{B}$ is the highest, $\mathrm{F}$ the lowest clearance). Taken from Galletti et al. (2003). 
Therefore the flow pattern transition which occurs for a RT when changing the impeller position is governed by two types of instability. The first one manifests as a random succession of double-loop regime, single-loop regime and transitional state over large time intervals. The second one is the instability encountered during the transitional state, characterised by a well-defined periodicity of the order of few seconds.

The exact nature of the clearance-related instabilities is not fully understood, but it is not likely to be related to the turbulence content of the flows, as the phenomenon is characterised by a single frequency even for the lowest Re range studied with the most viscous fluid, for which Re is around 5,200 and the corresponding flows should be mostly laminar. Some evidences as the increase of $\mathrm{f}^{\prime}$ with lowering $\mathrm{C} / \mathrm{T}$ (or increasing the impeller stream mean velocity by reducing the impeller blade thickness to diameter ratio $t_{b} / D$ ) may confirm that it is the interaction between the impeller discharge stream and the vessel base/walls to play a major role in the formation of such instability.

\subsection{Macro-instabilities}

Another kind of instability (see the right-hand side of the diagram of Fig. 3) manifests itself as large temporal and spatial variations of the flow superimposed to the mean flow patterns, thus such flow instabilities are called "macro-instabilities". On the basis of results achieved during our work and from other works in literature it was chosen to divide this kind of flow instability into two subgroups, because we think that there were two different underlying mechanisms driving such instabilities.

\subsubsection{Precessional macro-instabilities (P-MIs)}

The first subgroup comprehends flow instabilities which seem to be associated with a vortex moving about the shaft. The first evidence of this vortex was provided by Yianneskis et al. (1987) who noticed that the vortex motion produced large temporal and spatial fluctuations superimposed on the mean flow pattern. A similar vortex was also observed by Haam et al. (1992) cited earlier.

Precessional MIs were investigated by Nikiforaki et al. (2003), who used two different impellers (RT and PBT) having the same diameter $\mathrm{D}=\mathrm{T} / 3$ for $\operatorname{Re}>20,000$. The frequency of the macro-instabilities was found to be linearly related to the impeller speed with $f^{\prime}=f / N=$ 0.015-0.020, independently on impeller clearance and design. In a more recent work Nikiforaki et al. (2004) studied the effect of operating parameters on macro-instabilities. In particular they observed the presence of other frequencies varying from $\mathrm{f}^{\prime}=0.04-0.15$, as the Reynolds number was reduced.

Hartmann et al. (2004) performed a LES simulation of the turbulent flow ( $\operatorname{Re}=20,000$ and $30,000)$ in a vessel agitated with a $\mathrm{D}=\mathrm{T} / 3 \mathrm{RT}$ set at $\mathrm{C}=\mathrm{T} / 2$. The geometries of the vessel and impeller were identical to those used for the experiments of Nikiforaki et al. (2003). The simulation indicated the presence of a vortical structure moving round the vessel centreline in the same direction as the impeller. Such structure was observed both below and above the impeller (axial locations of $\mathrm{z} / \mathrm{T}=0.12$ and $\mathrm{z} / \mathrm{T}=0.88$ were monitored), however the two vortices were moving with a mutual phase difference. The frequency associated with the vortices was calculated to be $\mathrm{f}^{\prime}=0.0255$, therefore slightly higher than the $0.015-0.02$ reported by Nikiforaki et al. (2003). The authors concluded that this may encourage an improvement of the sub-scale grid and/or the numerical settings.

Importantly, the presence of a phase shift between the precessing vortices below and above the impeller was confirmed by the LDA experiments of Micheletti \& Yianneskis (2004). 
These authors used a cross-correlation method between data taken in the upper and lower parts of the vessel, and estimated a phase difference between the vortices in the two locations of approximately $180^{\circ}$.

The presence of the precessing vortex was assessed also in a solid-liquid system by the LES simulation of Derksen (2003).

Hasal et al. (2004) investigated flow instabilities with a Rushton turbine and a pitched blade turbine, both of $\mathrm{D}=\mathrm{T} / 3$ with the proper orthogonal decomposition analysis. They confirmed the presence of the precessing vortex, however they found different $\mathrm{f}^{\prime}$ values depending on the Re. In particular $\mathrm{f}^{\prime}$ values akin to those of Nikiforaki et al. (2003) were observed for high $\mathrm{Re}$, whereas higher values, i.e. $\mathrm{f}^{\prime}=0.06-0.09$ were found for low Re.

Galletti et al. (2004b) investigated macro-instabilities stemming from the precessional motion of a vortex about the shaft for different impellers, geometries and flow regimes. The authors confirmed that the P-MI frequency is linearly dependent on the impeller rotational speed, however they indicated that different values of the proportionality constant between MI frequency and impeller rotational speed were found for the laminar and turbulent flow regimes, indicating different behaviour of MIs depending on the flow Re (see Fig. 5a). For intermediate (transitional) regions two characteristic frequencies were observed, confirming the presence of two phenomena. In particular in the laminar flow region P-MIs occurred with a non-dimensional frequency $\mathrm{f}^{\prime}$ about 7-8 times greater than that observed for the turbulent region. This was proved for two RTs $(\mathrm{D} / \mathrm{T}=0.33$ and $0.41 \mathrm{RT})$ as well as for a $\mathrm{D} / \mathrm{T}$ $=0.46$ PBT. Thus the impeller design does not affect P-MIs for both laminar and turbulent regions. The impeller off-bottom clearance does not affect significantly the P-MI frequency for the Rushton turbine and the pitched blade turbine (see for instance Fig. 5b). However differences in the regions where P-MIs are stronger may be found, as for instance lower impeller clearances originated weaker P-MIs near the liquid surface.

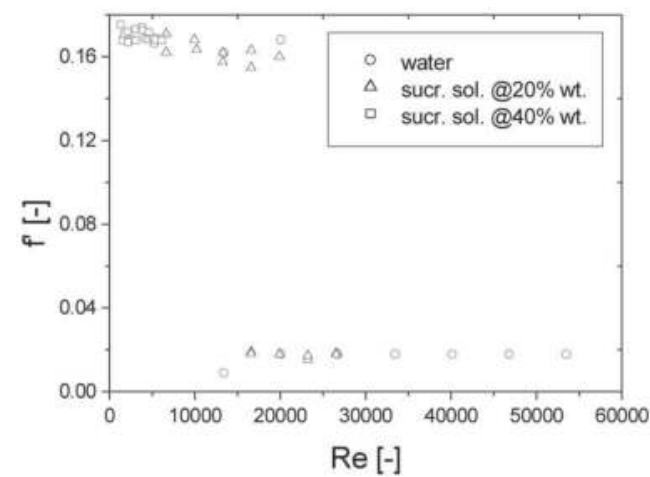

(a)

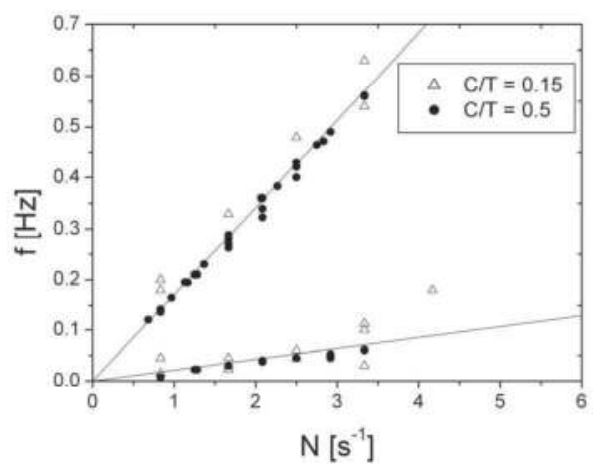

(b)

Fig. 5. (a) Non-dimensional macro-instability frequency as a function of the impeller Reynolds number. $\mathrm{RT}, \mathrm{D} / \mathrm{T}=0.41, \mathrm{C} / \mathrm{T}=0.5$. (b) Macro-instability frequency as a function of the impeller rotational speed for different clearances. RT, D/T $=0.41$. Galletti (2005).

Importantly, Galletti et al. (2004b) found that the MI frequency is affected by the impeller diameter. For the laminar regime a linear dependence of the non-dimensional macroinstability frequency on the impeller to tank diameter ratio was established: 


$$
f^{\prime}=a \cdot\left(\frac{D}{T}\right)+b
$$

A deep clarification of precessional MIs triggering mechanism in both laminar and turbulent regimes was provided by Ducci \& Yianneskis (2007) for a D = T/3 RT placed at $\mathrm{C}=\mathrm{T} / 2$. The authors used 2-point LDA and a 2-D PIV with a $13 \mathrm{kHz}$ camera. Through a vortex identification and tracking technique, the authors showed that P-MIs stem from a precessional vortex moving around the vessel axis with $f^{\prime}=0.0174$ for the turbulent regime. In laminar regime the frequency corresponding to a precession period was higher, of about $\mathrm{f}^{\prime}=0.13$. The slight differences on the frequencies with the work of Galletti et al. (2004b) may be imputed to the different spectral analysis. For instance in the vortex tracking method the frequency was evaluated from the time needed to a vortex to complete $360^{\circ}$, whereas the FFT analysis of Galletti et al. (2004b) covered several MI cycles. But importantly Ducci \& Yianneskis (2007) showed that in the laminar regime the vortex precessional motion was much closer to the axis than in turbulent regime (for which the vortex tends to stay rather far from the axis). In addition to that the authors showed a change in the flow pattern between the laminar and turbulent conditions, which affects the precessional MI frequency.

In a later work Ducci et al. (2008) investigated also the transitional regime showing the interaction between the two frequency instabilities $\left(f^{\prime}=0.1\right.$ and $f^{\prime}=0.02$ of the laminar and turbulent regime, respectively). They found that the two simultaneous instabilities are associated to two different types of perturbation of the main mean flow: an off-centering instability that results in a precession of the vortex core centre with a $\mathrm{f}^{\prime}=0.02$ and a stretching instability that induces an elongation of the vortex core along a direction that is rotating with $\mathrm{f}^{\prime}=0.1$ around the vessel axis. For higher $\mathrm{Re}$, the authors identified an interaction between the perturbations of the mean vortex core associated to $\mathrm{f}^{\prime}=0.02$ offcentering structures and $\mathrm{f}^{\prime}=0.04$ stretching/squeezing instability.

A deep investigation of precessional MIs was also carried out by the same group (Doulgerakis et al., 2011) for an axial impeller (PBT) with $\mathrm{D}=\mathrm{T} / 2$ placed at $\mathrm{C}=\mathrm{T} / 2$ with $\operatorname{Re}$ $=28,000$. The MI frequency distribution across the vessel indicated the presence of many frequencies reported before in literature. However the two dominant frequencies were $f^{\prime}=$ 0.1 and $f^{\prime}=0.2$. The POD analysis showed that the first mode can be seen as a radial offcenter perturbation of the mean flow that results in a precession of the vortex core around the impeller axis with $\mathrm{f}^{\prime}=0.1$. The second mode is an instability which stretches/squeezes the vortex core in a direction that is rotating with $\mathrm{f}^{\prime}=0.1$. Importantly also for the PBT, the higher frequency was exactly double than the lower one as for the RT case. This would be also in agreement with many spectral analysis reported in Galletti (2005) which showed the presence of an additional peak frequency about the double of the P-MI frequency.

Kilander et al. (2006) identified through LSP analysis of LDA data frequencies with $\mathrm{f}^{\prime}=0.025$ for the turbulent regime (thus in fully agreement with the work by Hartmaan et al., 2006) in a vessel agitated by a $\mathrm{D}=\mathrm{T} / 3 \mathrm{RT}$.

Lately, many other computational methods confirmed also the presence of precessional MIs. Nurtono et al. (2009) obtained from LES simulations a frequency $f^{\prime}=0.0125$ for a $D=T / 3$ RT placed at $\mathrm{C}=\mathrm{T} / 2$ for $\operatorname{Re}=40,000$.

The DNS simulations of Lavezzo et al. (2009) for an unbaffled vessel equipped with a 8blade paddle impeller indicated the presence of a spiralling vortex with $\mathrm{f}^{\prime}=0.162$ for $\mathrm{Re}=$ 1686. The application of Eq. [1] developed by Galletti et al. (2004b) to the above case would 
give a higher frequency $f^{\prime}=0.24$, however it should be pointed out that the equation was developed for baffled configurations.

\subsubsection{Jet impingement macro-instabilities (J-MIs)}

Other evidence of large temporal and spatial variations of the flow macro-instabilities have been reported in the last decade and they not always seem to be related to a precessional vortex.

Bruha et al. (1995) used a device called "tornadometer" to estimate the flow instabilities induced by a 6-bladed $45^{\circ} \mathrm{PBT}$ of $\mathrm{D}=0.3 \mathrm{~T}$ set at $\mathrm{C}=0.35 \mathrm{~T}$. The target was axially located above the impeller at $\mathrm{z} / \mathrm{C}=1.2$ and 1.4 and at radial distance equal to the impeller radius. The aforementioned authors found a linear relation between the instability frequency $f$ and the impeller rotational speed $\mathrm{N}$, according to $\mathrm{f}=-0.040 \mathrm{~N}+0.50$. In a later work (Bruha et al., $1996)$ the same authors reported a linear dependence of the MI frequency on $N\left(f^{\prime}=0.043\right.$ 0.0048 ) for Re values above 5,000. No flow-instabilities were noted for $\operatorname{Re}<200$ and an increase in $\mathrm{f}^{\prime}$ was observed for $200<\operatorname{Re}<5,000$.

Montes et al. (1997) studied with LDA the flow instabilities in the vicinity of the impeller. induced by a 6-bladed $45^{\circ}$ PBT of $\mathrm{D}=0.33 \mathrm{~T}$ set at $\mathrm{C}=0.35 \mathrm{~T}$ and observed different values for $\mathrm{f}^{\prime}$ depending on the Reynolds number: $\mathrm{f}^{\prime}=0.09$ for $\operatorname{Re}=1140$ and $\mathrm{f}^{\prime}=0.0575$ for $\operatorname{Re}=$ 75,000 . They suggested that macro-instabilities appear as the switching between one loop and two or many loops, taking place between the impeller and the free surface and they are able to alter this surface. This leads to different flow patterns in front of the baffles or between two adjacent baffles. The mechanism is complex and three-dimensional but the large vortices clearly appear in a regular way, with a well defined frequency. Hasal et al. (2000) used the proper orthogonal decomposition to analyse LDA data observed for a PBT and found $a f^{\prime}=0.087$ for $\operatorname{Re}=750$ and $\operatorname{Re}=1,200$, and a value of 0.057 for $\operatorname{Re}=75,000$. In addition they noticed that the fraction of the total kinetic energy carried by the flow instabilities (relative magnitude) varied with the location inside the stirred vessel, they being stronger in the central and wall regions below the impeller but weaker in the discharge flow from the impeller.

Myers et al. (1997) used digital PIV to investigate flow instabilities in a stirred tank equipped with two different impellers: a 4-bladed $45^{\circ} \mathrm{PBT}$ of $\mathrm{D}=0.35 \mathrm{~T}$ and a Chemineer HE-3 of $\mathrm{D}=0.39 \mathrm{~T}$. The PBT was set at $\mathrm{C}=0.46 \mathrm{~T}$ and $0.33 \mathrm{~T}$, whereas the Chemineer HE-3 was set at $C=0.33$ T. The Reynolds number was ranging between 6,190 and 13,100 . For the higher clearance, i.e. $\mathrm{C}=0.46 \mathrm{~T}$, the PBT showed flow fluctuations of about $40 \mathrm{~s}$ for an impeller rotational speed $\mathrm{N}=60 \mathrm{rpm}$, therefore $\mathrm{f}^{\prime}=0.025$. The same impeller set at the lower clearance, $C=0.33 \mathrm{~T}$, showed more stable flow fields, with not very clear peaks in the low frequency region of the spectra, at around $\mathrm{f}^{\prime}=0.07-0.011$. The Chemineer HE-3 impeller showed fluctuations of much longer periods than those of the PBT.

Roussinova et al. $(2000,2001)$ performed LDA measurements in two tank sizes $(T=0.24$ and $1.22 \mathrm{~m}$ ), using various impeller types, impeller sizes, clearances, number of baffles (2 and 4) and working fluids in fully turbulent regime. For a $45^{\circ} \mathrm{PBT}$ of $\mathrm{D}=\mathrm{T} / 2$ they observed a macro-instability non-dimensional frequency of $\mathrm{f}^{\prime}=0.186$. Such frequency was coherent as the PBT was set at $C=0.25 \mathrm{~T}$, and such a configuration was called "resonant" geometry, whereas a broad low frequency band was observed for different clearances. The same authors performed also a LES of the vessel stirred by a PBT and confirmed the above non dimensional frequency value. In a later work Roussinova et al. (2003) identified three possible mechanisms triggering the above flow instabilities: the impingement of the jet-like 
impeller stream on either the vessel walls or bottom, converging radial flow at the vessel bottom from the baffles and shedding of trailing vortices from the impeller blades. For the resonant geometry, the first mechanism coincided with the impingement of the discharge stream on the vessel corner, generating pressure waves reflected back towards the impeller. The impingement jet frequency was approached with a dimensional analysis based on the Strouhal number. We well denote such flow instabilities as jet impingement instabilities (JMIs). In a later investigation Roussinova et al. (2004) extended the analysis to different axial impellers. In such work the authors used the LSP method for the spectral analysis.

Paglianti et al. (2006) analysed literature data on MIs as well as comprehensive data obtained from measurements of wall pressure time series, and develop a simple model (based on a flow number) for predicting the MI frequency due to impinging jets (J-MI).

Also Galletti et al. (2005b) investigated flow instability for a PBT and detected a $\mathrm{f}^{\prime}=0.187$ (thus akin the Roussinova et al., 2003). Such instabilities were found to prevail in the region close to the impeller (just above it and below it in the discharged direction).

Nurtono et al. (2009) found a similar frequency $f^{\prime}=0.185$ from LES modelling of a $D=T / 3$ PBT placed at $\mathrm{C}=\mathrm{T} / 2$ at $\operatorname{Re}=40,000$.

The LES results on different impellers (DT, PBTD60, PBTD45, PBTD30 and HF) from Murthy \& Joshi (2008) showed the presence of J-MIs with $f^{\prime}=0.13-0.2$. Moreover they observed a frequency $\mathrm{f}^{\prime}=0.04-0.07$, which lies in between the precessional and the jet instability; such frequency was attributed to the interaction of precessing vortex instability with either the mean flow or jet/circulation instabilities.

Roy et al. (2010) investigated through both experimental (PIV) and numerical (LES) techniques, the flow induced by a PBT impeller at different Reynolds numbers $(\operatorname{Re}=44,000$, 88,000 and 132,000). They found low frequency flow instabilities with frequencies of about $\mathrm{f}^{\prime}$ $=0.2$. They could not resolve lower frequencies because of the short observation (due to computational cost of LES models) of their simulations. The authors showed changes in the three-dimensional flow pattern during different phases of the macro-instability cycle. They concluded that one mechanism driving flow instabilities was the interaction of the impeller jet stream with the tank baffles. The flow-instabilities were also observed to affect the dynamics of trailing edge vortices.

More recently Galletti \& Brunazzi (2008) investigated through LDA and flow visualisation the flow features of an unbaffled vessel stirred by an eccentrically positioned Rushton turbine. The flow field evidenced two main vortices: one departing from above the impeller towards the top of the vessel and one originating from the impeller blades towards the vessel bottom. The former vortex was observed to dominate all vessel motion, leading to a strong circumferential flow around it.

The frequency analysis of LDA data indicated the presence of well defined peaks in the frequency spectra of velocity recordings. In particular three characteristic frequencies were observed in different locations across the vessel: $f^{\prime}=0.105,0.155$ and 0.94 . Specifically, the $f^{\prime}$ $=0.155$ and 0.105 frequencies were related to the periodic movements of the upper and lower vortices' axis, respectively, which are also well visible from flow visualization experiments (see Fig. 6a and Fig. 6b, respectively). The $f^{\prime}=0.94$ frequency was explained by considering the vortical structure - shaft interaction, which occurs in eccentric configuration and leads to vortex shedding phenomena. The authors provided an interpretation based on the Strouhal number.

In a later work (Galletti et al., 2009) the effect of blade thickness $t_{b}$ was investigated, finding that for a thicker impeller $\left(t_{b} / D=0.05\right)$ the frequency of the upper vortex movement was 

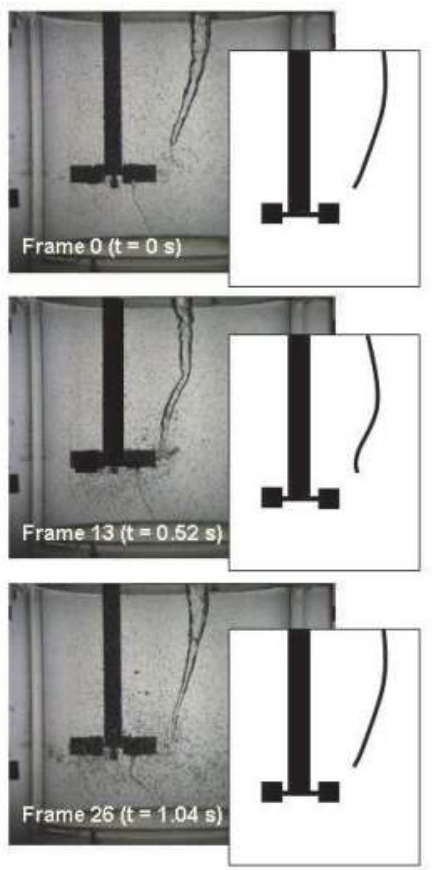

(a)
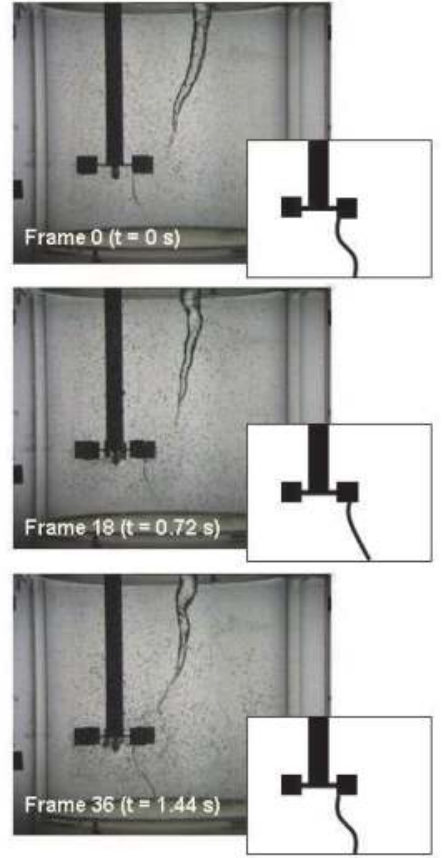

(b)

Fig. 6. Frames taken from flow visualisation experiments with sketches at $\mathrm{N}=400 \mathrm{rpm}$ (from Galletti \& Brunazzi, 2008). Unbaffled vessel, RT, eccentricity E/T $=0.21, C / T=0.33$, , $\mathrm{D} / \mathrm{T}=0.33, \mathrm{t}_{\mathrm{b}} / \mathrm{D}=0.01$.

lower, i.e. $f^{\prime}=0.143$ than for the thinner one $\left(f^{\prime}=0.155\right.$ for $\left.t_{b} / D=0.01\right)$. The origin of the above instabilities in not fully clarified. The frequencies are one order of magnitude higher than the P-MIs frequencies. The values of $\mathrm{f}^{\prime}$ found are more similar to frequencies typical of J-MIs. Actually the eccentric position of the shaft and the consequently reduced distance between the impeller blade tip and the vessel boundaries, is likely to enhance the strength of the impeller discharged stream - wall interaction. In such a case, resulting flow instabilities will show a frequency which is expected to increase with increasing the velocity of the impeller discharged stream (see the flow-instability analysis in terms of pumping number by Paglianti et al., 2006, and/or peak velocity by Roussinova et al., 2003), thus with decreasing the blade thickness (Rutherford et al., 1996b).

\section{Effect of flow instabilities}

Flow instabilities may affect mixing operations in mechanically agitated vessels in different manners.

Since the energetic content of J-MIs may be significant, they can exert strong forces on the solid surfaces immersed in the stirred tank, i.e. the shaft, baffles, heating and cooling coils, etc. (Hasal et al., 2004). These forces may cause mechanical failure of the equipment and therefore they should be taken into account in the design of industrial-scale stirred vessels. 
However except for such drawbacks, MIs may be beneficially utilized to improve mixing, provided that their phenomenology is well understood.

It has been proved than flow instabilities in stirred vessels can have a direct effect on overall parameters, which are fundamental for the design practice. The different studies on the change of circulation pattern (mentioned in section 4.1) have evidenced that such change is accompanied by a change of power number. In case of solid suspension, changes in the Njs is observed. Thus the knowledge of parameters affecting the circulation change may help optimising solid-liquid operations. Moreover, the heat flux studies of Haam et al. (1992) showed that precessional MIs may induce a variation of the heat transfer coefficient up to $68 \%$ near the surface.

Macro-instabilities may have beneficial implications for mixing process operation and efficiency as such flow motions can enhance mixing through mean-flow variations. For example, the associated low-frequency, high-amplitude oscillatory motions in regions of low turbulence in a vessel, have the capability of transporting substances fed to a mixing process over relatively long distances, as demonstrated by Larsson et al. (1996). These authors measured glucose concentration in a cultivation of Saccharomyces Cerevisiae and observed fluctuations of glucose concentration which were more pronounced as the feed was located in a stagnant area rather than in the well-mixed impeller area. Therefore flow instabilities may help destroying segregated zones inside the tank. Ducci \& Yianneskis (2007) showed that the mixing time could be reduced even by $30 \%$ if the tracer is inserted at or near the MI vortex core. Houcine et al. (1999) reported with LIF a feedstream jet intermittency in a continuous stirred tank reactor due to MIs. Recently also Galletti et al. (2009) observed from decolourisation experiments in an eccentrically agitated unbaffled vessel that the flow instability oscillations help the transport of reactants far away if these are fed in correspondence of the vortices shown in Fig. 6.

Subsequently MIs have similar effects to those reported for laminar mixing in stirred tanks by Murakami et al. (1980), who observed that additional raising and lowering of a rotating impeller produced unsteady mean flow motions that either destroyed segregated regions or prevented them from forming, and could produce desired mixing times with energy savings of up to $90 \%$ in comparison to normal impeller operation. Later Nomura et al. (1997) observed that the reversal of the rotational direction of an impeller could also decrease mixing times as the additional raising or lowering of the impeller.

For a solid-liquid system (solid volume fractions up to $3.6 \%$ ) agitated by a D = T/3 RT in turbulent regime $(\operatorname{Re}=100,000$ and 150,000) Derksen (2003) showed that the precessing vortex may help the resuspension of particles lying on the bottom of the tank, thus enhancing the mass transfer.

Guillard et al. (2000a) carried out LIF experiments on a stirred tank equipped with two RT observing large time scale oscillations of the concentration, induced by an interaction between the flows from the impeller and a baffle. They argued that circulation times can be altered when the flow direction changes, the turbulence levels measured with stationary probes can be significantly broadened and thus can provide an erroneous interpretation of the true levels of turbulence in a tank, and mixing in otherwise quiescent regions can be significantly enhanced due to the presence of flow variations (Guillard et al., 2000b). Knoweledge of true levels of turbulence is needed for the optimum design of micro-mixing operations (as in cases of chemical reactions). Also Nikiforaki et al. (2003) observed that PMIs can broaden real turbulence levels up to $25 \%$ for a PBT. 
Actually the problem is rather complex as Galletti et al. (2005b) as well other investigators (e.g. Ducci \& Yianneskis, 2007, Roussinova et al., 2004) showed that different kinds of macro-instabilities may be present simultaneously in stirred vessels. For instance Galletti et al. (2005b) studied simultaneously with 2-point LDA the combined effect of precessional MIs and flow instabilities stemming from impeller clearance variations (CIs) in different regions of a vessel stirred with a RT. Table 1 summarizes the flow instability characteristics. The authors removed from the total energetic content of a LDA signal, the contribution of blade passage, P-MIs and CIs, evaluating the real turbulent energy. They found that the occurrence and energetic content of P-MIs and CIs depend on both measurement location and flow regime. In particular, near the vessel surface P-MIs are stronger, with energetic contents that reach $50 \%$ of the turbulent energy, meaning that they can broaden turbulence levels up to $22 \%$. In the vicinity of the impeller the energetic content of the P-MIs is smaller, whereas CIs contribute strongly to the fluid motion with average energetic contents of about $21 \%$ of the turbulent energy for the transitional regime. Results are summarised in Table 2.

\begin{tabular}{|c|c|c|}
\hline \multicolumn{3}{|c|}{ Rushton turbine } \\
\hline Flow instability & CIs & P-MIs \\
\hline How they manifest & change in circulation & $\begin{array}{l}\text { large temporal and spatial } \\
\text { fluctuation superimposed on } \\
\text { the mean flow pattern }\end{array}$ \\
\hline Impeller/vessel configuration & $\begin{array}{c}\text { specific configuration } \\
(C / T=0.17-0.2 \text { with } D / T= \\
0.33)\end{array}$ & $\begin{array}{c}\text { several configurations } \\
\text { (different impeller types } D / T \text {, } \\
C / T \text { ) }\end{array}$ \\
\hline Temporal appearance & intermittently present & continuously present \\
\hline Non-dimensional frequency & $f^{\prime}=0.13$ & $f^{\prime}=0.015$ \\
\hline Possible origin & $\begin{array}{l}\text { interaction between impeller } \\
\text { discharged stream and vessel } \\
\text { base/walls }\end{array}$ & $\begin{array}{l}\text { precessional motion of a } \\
\text { vortex about the shaft }\end{array}$ \\
\hline
\end{tabular}

Table 1. Characteristics of CIs and MIs investigated with the Rushton turbine. Galletti (2005).

\begin{tabular}{ccccc}
\hline & \multicolumn{2}{c}{ Near the surface } & \multicolumn{2}{c}{ Impeller region } \\
\hline & $E_{M I} / E_{T U R}$ & $E_{\mathrm{CI}} / \mathrm{E}_{\mathrm{TUR}}$ & $E_{M I} / E_{T U R}$ & $E_{\mathrm{CI}} / E_{T U R}$ \\
\hline double-loop & up to $50 \%$ & $\sim 4 \%$ & $\sim 5 \%$ & $\sim 3 \%$ \\
\hline transitional state & up to $25 \%$ & up to $25 \%$ & negligible & $\sim 21 \%$ \\
\hline single-loop & $\sim 12 \%$ & $\sim 3 \%$ & negligible & negligible \\
\hline
\end{tabular}

Table 2. Relative energy of MIs and CIs with respect to the turbulent energy for the double-, single- and transitional patterns. Galletti (2005). 
A similar analysis was carried out for a PBT: in this case the P-MIs and J-MIs were studied (see Table 3). The authors found the presence of both instabilities, indicating that the occurrence and magnitude, i.e. energetic content, of MIs and JIs vary substantially from one region of a vessel to another. P-MIs affect strongly the region of the vessel near the surface and around the shaft, whereas the bulk of the vessel is dominated more by J-MIs generated from the interaction of the impeller discharged stream and the vessel boundaries. J-MIs are also stronger upstream of the baffles and near the walls, which may confirm their origin. Table 4 reports the energetic contribution of the different macro-instabilities at different axial location in the vessel.

\begin{tabular}{ccc}
\hline \multicolumn{3}{c}{ Pitched Blade Turbine } \\
\hline Flow instability & J-MIs & P-MIs \\
\hline How they manifest & $\begin{array}{c}\text { large temporal and spatial } \\
\text { fluctuation superimposed on } \\
\text { the mean flow pattern }\end{array}$ & $\begin{array}{c}\text { large temporal and spatial } \\
\text { fluctuation superimposed on } \\
\text { the mean flow pattern }\end{array}$ \\
\hline Impeller/vessel configuration & $\begin{array}{c}\text { specific configuration } \\
(C / T=0.25 \text { with } D / T=0.5)\end{array}$ & $\begin{array}{c}\text { several configurations } \\
\text { different impeller types } D / T, \\
C / T)\end{array}$ \\
\hline Temporal appearance & continuously present & continuously present \\
\hline Non-dimensional frequency & $f^{\prime}=0.186$ & $f^{\prime}=0.015$ \\
\hline Possible origin & $\begin{array}{c}\text { interaction between impeller } \\
\text { discharged stream and vessel } \\
\text { base/walls }\end{array}$ & $\begin{array}{c}\text { precessional motion of a } \\
\text { vortex about the shaft }\end{array}$ \\
\hline
\end{tabular}

Table 3. Characteristics of JIs and MIs investigated with the pitched blade turbine. Galletti (2005).

\begin{tabular}{ccccc}
\hline & \multicolumn{2}{c}{ P-MIs } & \multicolumn{2}{c}{ J-MIs } \\
\hline $\begin{array}{c}\text { Location of the } \\
\text { horizontal plane }\end{array}$ & $\begin{array}{c}\text { Max } \\
E_{M I} / E_{T U R}\end{array}$ & $\begin{array}{c}\text { Average } \\
E_{M I} / E_{T U R}\end{array}$ & $\begin{array}{c}\text { Max } \\
E_{I I} / E_{T U R}\end{array}$ & $\begin{array}{c}\text { Average } \\
E_{I I} / E_{T U R}\end{array}$ \\
\hline$z / T=0.05$ & $1.9 \%$ & $5.7 \%$ & $2.7 \%$ & $6.3 \%$ \\
\hline$z / T=0.6$ & $6.2 \%$ & $12 \%$ & $10.1 \%$ & $20 \%$ \\
\hline$z / T=0.93$ & $14.6 \%$ & $39.8 \%$ & $1.7 \%$ & $7 \%$ \\
\hline
\end{tabular}

Table 4. Average and maximum relative energy of MIs and JIs with respect to the turbulent energy, for different horizontal planes. Galletti (2005).

For the eccentric agitation in an unbaffled vessel, Galletti \& Brunazzi (2008) showed that the flow instability related to the movement of the two vortices described in section 4.2.2. was very strong, as its energetic contribution was evaluated to be as high as $52 \%$ of the turbulent kinetic energy. Also the shedding vortices from flow-shaft interaction considerably affected the turbulence levels (energetic contribution of $82 \%$ ), hence they should be considered in evaluating the micro-mixing scales. 


\section{References}

Armenante, P.M. \& Nagamine, E.U. (1998). Effect of low off-bottom impeller clearance on the minimum agitation speed for complete suspension of solids in stirred tanks. Chemical Engineering Science, Vol. 53, pp. 1757-1775.

Assirelli, M.; Bujalski, W.; Eaglesham, A. \& Nienow, A.W. (2005). Intensifying micromixing in a semi-batch reactor using a Rushton turbine. Chemical Engineering Science, Vol. 60, pp.2333-2339.

Bakker, A.; Fasano, J.B. \& Myers, K.J. (1998). Effects of flow pattern on the solid distribution in a stirred tank, Published in "The online CFM Book" at http://www.bakker.org.cfm.

Bakker, A.; La Roche, R.D.; Wang, M. \& Calabrese, R. (1997). Sliding mesh simulation of laminar flow in stirred reactors. Transactions IChemE, Chemical Engineering Research and Design, Vol. 75, pp. 42-44.

Baldi, G.; Conti, R. \& Alaria, E. (1978). Predicting the minimum suspension speeds in agitated tanks. Chemical Engineering Science, Vol. 33, pp. 21-25.

Berkooz, G.; Holmes, P. \& Lumley, J.L. (1993). The proper orthogonal decomposition in the analysis of turbulent flows. Annual Review of Fluid Mechanics, Vol. 25, pp. 539-576.

Bruha, O.; Fort, I. \& Smolka, P. (1995). Phenomenon of turbulent macro-instabilities in agitated systems. Collection of Czechoslovak Chemical Communications, Vol. 60, pp. 8594.

Bruha, O.; Fort, I.; Smolka, P. \& Jahoda, M. (1996). Experimental study of turbulent macroinstabilities in an agitated system with axial high-speed impeller and with radial baffles. Collection of Czechoslovak Chemical Communications, Vol. 61, pp. 856867.

Conti, R.; Sicardi, S. \& Specchia, V. (1981). Effect of the stirrer clearance on suspension in agitated vessels. Chemical Engineering Journal, Vol. 22, pp. 247-249.

Cooley, J.W. \& Tukey, J.W. (1965). An algorithm for the machine calculation of complex Fourier series. Mathematical Computation, Vol. 19, pp. 297-301.

Daubechies, I. (1990). The Wavelet transform time-frequency localization and signal analysis. IEEE Transactions on Information Theory, Vol. 36, pp. 961-1005.

Derksen, J.J. (2003). Numerical simulation of solids suspension in a stirred tank, AIChE Journal. Vol. 49, pp. 2700-2714.

Distelhoff, M.F.W.; Laker, J.; Marquis, A.J. \& Nouri, J. (1995). The application of a straingauge technique to the measurement of the power characteristics of 5 impellers. Experiments in Fluids, Vol. 20, pp. 56-58.

Doulgerakis, Z.; Yianneskis, M.; Ducci, A. (2011). On the interaction of trailing and macroinstability vortices in a stirred vessel-enhanced energy levels and improved mixing potential. Chemical Engineering Research and Design, Vol. 87, pp. 412-420.

Ducci, A. \& Yianneskis, M. (2007). Vortex tracking and mixing enhancement in stirred processes. AIChE Journal, Vol. 53, pp. 305-315.

Ducci, A.; Doulgerakis, Z. \& Yianneskis, M. (2008). Decomposition of flow structures in stirred reactors and implications for mixing enhancement. Industrial and Engineering Chemistry Research, Vol. 47, pp. 3664-3676.

Galletti, C. \& Brunazzi, E. (2008). On the main flow features and instabilities in an unbaffled vessel agitated with an eccentrically located impeller. Chemical Engineering Science, Vol 63, pp. 4494-4505 
Galletti, C. (2005). Experimental analysis and modeling of stirred vessels. Ph.D. thesis, University of Pisa, Pisa, Italy.

Galletti, C.; Brunazzi, E.; Pintus, S.; Paglianti, A. \& Yianneskis, M. (2004a). A study of Reynolds stresses, triple products and turbulence states in a radially stirred tank with 3-D laser anemometry. Transactions IChemE, Chemical Engineering Research and Design, Vol. 82, pp. 1214-1228.

Galletti, C.; Brunazzi, E.; Yianneskis, M. \& Paglianti, A. (2003). Spectral and Wavelet analysis of the flow pattern transition with impeller clearance variations in a stirred vessel. Chemical Engineering Science, Vol. 58, pp. 3859-3875.

Galletti, C.; Lee, K.C.; Paglianti, A. \& Yianneskis, M. (2005a). Flow instabilities associated with impeller clearance changes in stirred vessels. Chemical Engineering Communications, Vol. 192, pp. 516-531.

Galletti, C.; Lee, K.C.; Paglianti, A. \& Yianneskis, M. (2004b). Reynolds number and impeller diameter effects on instabilities in stirred vessels, AIChE Journal, Vol. 50, 2050-2063.

Galletti, C.; Paglianti, A. \& Yianneskis, M. (2005b). Observations on the significance of instabilities, turbulence and intermittent motions on fluid mixing processes in stirred reactors. Chemical Engineering Science, Vol. 60, pp. 2317-2331.

Galletti, C.; Pintus, S. \& Brunazzi, E. (2009). Effect of shaft eccentricity and impeller blade thickness on the vortices features in an unbaffled vessel. Chemical Engineering Research and Design, Vol. 87, pp. 391-400.

Guillard, F.; Trägårdh, C. \& Fuchs, L. (2000a). A study of turbulent mixing in a turbineagitated tank using a fluorescence technique. Experiments in Fluids, Vol. 28, pp. 225235.

Guillard, F.; Trägårdh, C. \& Fuchs, L. (2000b). A study on the instability of coherent mixing structures in a continuously stirred tank. Chemical Engineering Science, Vol. 55, pp. 5657-5670.

Haam, S.; Brodkey, R.S. \& Fasano, J.B. (1992). Local heat-transfer in a mixing vessel using heat-flux sensors. Industrial and Engineering Chemistry Research, Vol. 31, 1384-1391.

Hartmann, H.; Derksen, J.J. \& van den Akker H.E.A. (2006). Numerical simulation of a dissolution process in a stirred tank reactor. Chemical Engineering Science. Vol 61 , pp. $3025-3032$

Hartmann, H.; Derksen, J.J. \& van den Akker, H.E.A. (2004). Macroinstability uncovered in a Rushton turbine stirred tank by means of LES. AIChE Journal, Vol. 50, pp. 23832393.

Hasal, P.; Fort, I. \& Kratena, J. (2004). Force effects of the macro-instability of flow pattern on radial baffles in a stirred vessel with pitched-blade and Rushton turbine impeller. Transactions IChemE, Chemical Engineering Research and Design, Vol. 82, pp. 12681281.

Hasal, P.; Montes, J.L.; Boisson, H.C. \& Fort, I. (2000). Macro-instabilities of velocity field in stirred vessel: detection and analysis. Chemical Engineering Science, Vol. 55, pp. 391401.

Hockey, R. M. \& Nouri, M. (1996). Turbulent flow in a baffled vessel stirred by a $60^{\circ}$ pitched blade impeller. Chemical Engineering Science, Vol. 51, pp. 4405-4421.

Hockey, R.M. (1990). Turbulent Newtonian and non-Newtonian flows in a stirred reactor, Ph.D. thesis, Imperial College, London. 
Houcine, I.; Plasari, E.; David, R. \& Villermaux, J. (1999). Feedstream jet intermittency phenomenon in a continuous stirred tank reactor. Chemical Engineering Journal, Vol. 72, pp. 19-29.

Ibrahim, S. \& Nienow, A.W. (1995). Power curves and flow patterns for a range of impellers in Newtonian fluid: $40<R e<5 \times 10^{5}$. Transactions IChemE, Chemical Engineering Research and Design, Vol. 73, pp. 485-491.

Ibrahim, S. \& Nienow, A.W. (1996). Particle suspension in the turbulent regime: the effect of impeller type and impeller/vessel configuration. Transactions IChemE, Chemical Engineering Research and Design, Vol. 74, pp. 679-688.

Jaworski, Z.; Nienow, A.W.; Koutsakos, E.; Dyster, K. \& Bujalski, W. (1991). An LDA study of turbulent flow in a baffled vessel agitated by a pitched blade turbine. Transactions IChemE, Chemical Engineering Research and Design, Vol. 69, pp. 313-320.

Kilander, J; Svensson, F.J.E. \& Rasmuson, A. (2006). Flow instabilities, energy levels, and structure in stirred tanks. AIChE Journal, Vol. 52, pp. 3049-4051.

Kresta, S.M. \& Wood, P.E. (1993). The mean flow field produced by a $45^{\circ}$ pitched-blade turbine: changes in the circulation pattern due to off bottom clearance. Canadian Journal of Chemical Engineering, Vol. 71, pp. 42-53.

Larsson G.; To"rnkvist M.; Ståhl Wernersson E.; Tra"gårdh ,C.; Noorman, H. \& Enfors, S.O. (1996). Substrate gradients in bioreactors: origin and consequences. Bioprocess Engineering, Vol. 14, pp. 281-289.

Lavezzo, V.; Verzicco, R. \& Soldati, A. (2009). Ekman pumping and intermittent particle resuspension in a stirred tank reactor. Chemical Engineering Research and Design. Vol. 87, pp. 557-564.

Lomb, N.R. (1976). Least-squares frequency analysis of unequally spaced data, Astrophysics and Space Science. Vol. 39, pp. 447-462.

Mao, D.; Feng, L.; Wang, K. \& Li, Y. (1997). The mean flow generated by a pitched-blade turbine: changes in the circulation pattern due to impeller geometry, Canadian Journal of Chemical Engineering, Vol. 75, pp. 307-316.

Micheletti, M. \& Yianneskis, M. (2004). Precessional flow macro-instabilities in stirred vessels: study of variations in two locations through conditional phase-averaging and cross-correlation approaches, Proceedings $11^{\text {th }}$ International Symposium on Applications of Laser Techniques to Fluid Mechanics, Lisbon, Portugal.

Montante, G.; Lee, K.C.; Brucato, A. \& Yianneskis, M. (1999). Double- to single- loop flow pattern transition in stirred vessels. Canadian Journal of Chemical Engineering, Vol. 77, pp. 649-659.

Montes, J.L.; Boisson H.C.; Fort, I. \& Jahoda, M. (1997). Velocity field macro-instabilities in an axially agitated mixing vessel. Chemical Engineering Journal, Vol. 67, pp. 139-145.

Murakami, Y.; Hirose, T.; Yamato, T.; Fujiwara, H. \& Ohshima, M. (1980). Improvement in mixing of high viscosity liquid by additional up-and-down motion of a rotating impeller. Journal of Chemical Engineering of Japan, Vol. 13, pp. 318-323.

Murthy, B.N. \& Joshi, J.B. (2008). Assessment of standard k-ع, RSM and LES turbulence models in a baffled stirred vessel agitated by various impeller designs. Chemical Engineering Science, Vol. 63, pp. 5468-5495

Myers, K.J.; Bakker, A. \& Corpstein, R.R. (1996). The effect of flow reversal on solids suspension in agitated vessels. Canadian Journal of Chemical Engineering 74, pp. 10281033. 
Myers, K.J.; Ward, R.W. \& Bakker, A. (1997). A digital particle image velocimetry investigation of flow field instabilities of axial-flow impellers. Journal of Fluid Engineering, Vol. 119, pp. 623-632.

Nienow, A. W. (1968). Suspension of solid particles in turbine agitated baffled vessels. Chemical Engineering Science, Vol. 23, pp. 1453-1459.

Nikiforaki, L.; Montante, G.; Lee, K.C.; Yianneskis, M. (2003). On the origin, frequency and magnitude of macro-instabilities of the flows in stirred vessels. Chemical Engineering Science, Vol. 58, pp. 2937-2949.

Nikiforaki, L.; Yu, J.; Baldi, S.; Genenger, B.; Lee, K.C.; Durst, F. \& Yianneskis, M. (2004). On the variation of precessional flow instabilities with operational parameters in stirred vessels. Chemical Engineering Journal, Vol. 102, pp. 217-231.

Nomura, T.; Uchida, T. \& Takahashi, K. (1997). Enhancement of mixing by unsteady agitation of an impeller in an agitated vessel. Journal of Chemical Engineering of Japan, Vol. 30, pp. 875-879.

Nouri, J.M. \& Whitelaw, J.H. (1990). Flow characteristics of stirred reactors with Newtonian and non-Newtonian fluids. AIChE Journal, Vol. 36, pp. 627-629.

Nurtono T.; Setyawan; H. Altway A. \& Winardi, S. (2009) Macro-instability characteristic in agitated tank based on flow visualization experiment and large eddy simulation. Chemical Engineering Research and Design, Vol. 87, pp. 923-942.

Paglianti, A.; Montante, G. \& Magelli, F. (2006). Novel experiments and a mechanistic model for macroinstabilities in stirred tanks. AIChE Journal, Vol. 52, pp. 426-437.

Roussinova, V.T.; Grgic, B. and Kresta, S.M. (2000). Study of macro-instabilities in stirred tanks using a velocity decomposition technique. Transactions IChemE, Chemical Engineering Research and Design, Vol. 78, 1040-1052.

Roussinova, V.T.; Kresta, S.M. \& Weetman, R. (2003). Low frequency macroinstabilities in a stirred tank: scale-up and prediction based on large eddy simulations. Chemical Engineering Science, Vol. 58, pp. 2297-2311.

Roussinova, V.T.; Kresta, S.M. \& Weetman, R. (2004). Resonant geometries for circulation pattern macroinstabilities in a stirred tank. AIChE Journal, Vol. 50, pp. 2986-3005.

Roussinova, V.T.; Weetman, R. \& Kresta, S.M. (2001). Large eddy simulation of macroinstabilities in a stirred tank with experimental validation at two scales. North American Mixing Forum 2001.

Roy, S.; Acharya, S. \& Cloeter, M. (2010). Flow structure and the effect of macro-instabilities in a pitched-blade stirred tank. Chemical Engineering Science, Vol. 65, pp. 3009-3024.

Rutherford, K.; Lee, K.C.; Mahmoudi, S.M.S. \& Yianneskis, M. (1996a). Hydrodynamic characteristics of dual Rushton impeller stirred vessels. AIChE Journal, Vol. 42, pp. 332-346.

Rutherford, K.; Mahmoudi, S.M.S.; Lee, K.C. \& Yianneskis, M. (1996b). The Influence of Rushton impeller blade and disk thickness on the mixing characteristics of stirred vessels. Transactions IChemE, Chemical Engineering Research and Design, Vol. 74, pp. 369-378.

Scargle, J. (1982). Studies in astronomical time series analysis. II Statistical aspect of spectral analysis of unevenly spaced data. Astrophysical Journal, Vol. 263, pp. 835-853.

Schäfer, M.; Yianneskis, M.; Wächter, P. \& Durst, F. (1998). Trailing vortices around a $45^{\circ}$ pitched-blade impeller. AIChE Journal, Vol. 44, pp. 1233-1246. 
Sharma, R.N. \& Shaikh, A.A. (2003). Solids suspension in stirred tanks with pitched blade turbines. Chemical Engineering Science, Vol. 58, pp. 2123-2140.

Tatterson, G.B. (1991). Fluid mixing and gas dispersion in agitated tanks, Mcgraw-Hill Inc., New York.

Tiljander, P. \& Theliander, H. (1993). Power-consumption and solid suspension in completely filled vessels. Chemical Engineering Communications, Vol. 124, pp. 1-14.

Torré, J.P.; Fletcher, D.F; Lasuye T. \& Xuereb, C. (2007) Single and multiphase CFD approaches for modelling partially baffled stirred vessels: Comparison of experimental data with numerical predictions. Chemical Engineering Science, Vol. 62, pp. 6246-6262

Torrence, C. \& Compo, P. (1998). A practical guide to Wavelet analysis. Bulletin of the American Meteorological Society, Vol. 79, pp. 61-78.

Yianneskis, M.; Popiolek, Z. \& Whitelaw, J.H. (1987). An experimental study of the steady and unsteady flow characteristics of stirred reactors. Journal of Fluid Mechanics, Vol. 175, pp. 537-555.

Zwietering, T.N. (1958). Suspending of solid particles in liquid by agitators. Chemical Engineering Science, Vol. 8, pp. 244-253. 


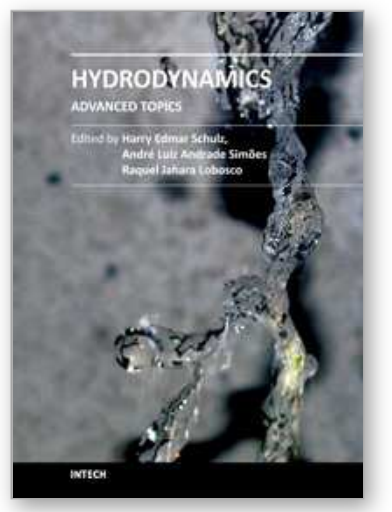

\author{
Hydrodynamics - Advanced Topics
}

Edited by Prof. Harry Schulz

ISBN 978-953-307-596-9

Hard cover, 442 pages

Publisher InTech

Published online 22, December, 2011

Published in print edition December, 2011

The phenomena related to the flow of fluids are generally complex, and difficult to quantify. New approaches considering points of view still not explored - may introduce useful tools in the study of Hydrodynamics and the related transport phenomena. The details of the flows and the properties of the fluids must be considered on a very small scale perspective. Consequently, new concepts and tools are generated to better describe the fluids and their properties. This volume presents conclusions about advanced topics of calculated and observed flows. It contains eighteen chapters, organized in five sections: 1) Mathematical Models in Fluid Mechanics, 2) Biological Applications and Biohydrodynamics, 3) Detailed Experimental Analyses of Fluids and Flows, 4) Radiation-, Electro-, Magnetohydrodynamics, and Magnetorheology, 5) Special Topics on Simulations and Experimental Data. These chapters present new points of view about methods and tools used in Hydrodynamics.

\title{
How to reference
}

In order to correctly reference this scholarly work, feel free to copy and paste the following:

Chiara Galletti and Elisabetta Brunazzi (2011). Flow Instabilities in Mechanically Agitated Stirred Vessels, Hydrodynamics - Advanced Topics, Prof. Harry Schulz (Ed.), ISBN: 978-953-307-596-9, InTech, Available from: http://www.intechopen.com/books/hydrodynamics-advanced-topics/flow-instabilities-in-mechanicallyagitated-stirred-vessels

\section{INTECH}

open science | open minds

\section{InTech Europe}

University Campus STeP Ri

Slavka Krautzeka 83/A

51000 Rijeka, Croatia

Phone: +385 (51) 770447

Fax: +385 (51) 686166

www.intechopen.com

\section{InTech China}

Unit 405, Office Block, Hotel Equatorial Shanghai

No.65, Yan An Road (West), Shanghai, 200040, China

中国上海市延安西路65号上海国际贵都大饭店办公楼 405 单元

Phone: +86-21-62489820

Fax: $+86-21-62489821$ 
(C) 2011 The Author(s). Licensee IntechOpen. This is an open access article distributed under the terms of the Creative Commons Attribution 3.0 License, which permits unrestricted use, distribution, and reproduction in any medium, provided the original work is properly cited. 